\title{
A Localized Region based Active Contour Method for Image Segmentation using Dynamic Threshold
}

\author{
Gajendra Kumar Sharma \\ Jaipur Institute of Technology \\ Kalwar Jaipur
}

\author{
Shashi Sharma \\ Jaipur Institute of Technology \\ Kalwar Jaipur
}

\begin{abstract}
Image segmentation is a fundamental image preparing approach this is utilized to research what is the component of the image graph. Image segmentation is utilized to part an image into various "huge" segments. The paper basically offers with surface segmentation while actualities pixel end up noticeably given mixed and blended error diminished so it could be one of the issues to manage likewise. It will be fundamentally constructed absolutely in light of segmentation of substance basically construct generally in light of the edge and we will show it in implementation how results alterations as consistent with a substitute in threshold values. Everything about pixels in a round is commensurable with the record to a couple of appropriate or registered resources, quiet with shading, intensity or surface Graph lessen calculations are effectively connected to a broad scope of issues in innovative and farsighted and images. Here we utilized this dynamic threshold procedure to improve the image segmentation inconvenience. What's more, we were given achievement results in apportioning an in the image. In this paper, we utilize the standardized reduce method technique to do the segmentation of a image graph. In this method; we utilize a computational system in view of the threshold esteem changes progressively and eigenvectors to get an improved sectioned image. We have completed this strategy to portion the static images. The experiment on probe images demonstrate that: our proposed approach can decrease the quantity of iterations, which prompts a huge diminishment in the computational cost while accomplishing comparative levels of accuracy. The approach additionally functions admirably when connected to image segmentation.
\end{abstract}

\section{Keywords}

Image segmentation, Edge-Based Segmentation, Clustering method, gradient

\section{INTRODUCTION \\ 1.1 Segmentation}

We all in all understand that each image is a course of action of pixels. Likewise, distributing those pixels on the introduce of the equivalent properties they have is called division isolating a image into sub allocates the preface of some near qualities like shading, drive and surface is called image division. The goal of division is to change the depiction of a image into something more critical and less difficult to analyze. Image division is normally used to discover things and limits that is lines, curves, and so on in images.

In image division image is isolated into a couple of regions and in that district each pixel is tantamount with respect to a segment of the trademark, for instance, the shading, compel, or surface. Neighboring locale are basically exceptional concerning the same characteristic(s).

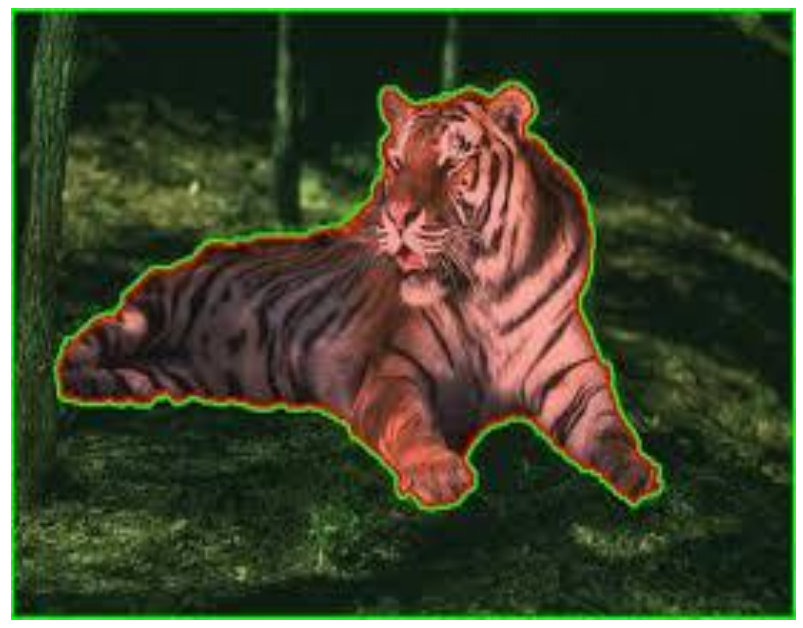

Fig 1: Segmented image

Pixels at which the power of a image changes unexpectedly are called edge pixels. Edges are set of associated edge pixels. An edge basically differentiates between two unmistakably extraordinary areas. It is the most widely recognized approach for location of dark level intermittent in a image.

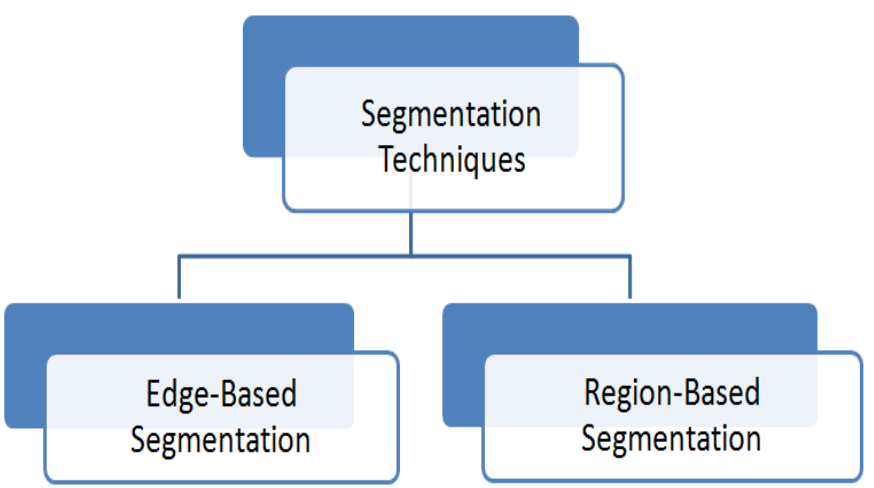

Fig 2: Image Segmentation approach

\subsection{Edge-Based Segmentation}

Division Methods in view of Discontinuity find for sudden changes in the power esteem. These strategies are called as Edge or Boundary based techniques. Edge identification is the issue of major significance in image examination. Edge discovery procedures are for the most part utilized for discovering discontinuities in dark level images.

\subsection{Region-Based Segmentation}

Area based strategies are based coherence. These procedures isolate the whole image into sub areas relying upon a few standards like every one of the pixels in one locale must have 
a similar dim level. District construct systems depend in light of basic examples in force values inside a bunch of neighboring pixels.

\subsection{Watershed Transformation}

Watershed Transformation has a place with the class of the area based likenesses. Watershed model is a numerical morphological approach and gets its similarity from a genuine surge circumstance [8]. It changes image into an angle image. At that point, image is viewed as a geological surface where dim qualities are considered as height of the surface at that area. At that point, flooding process begins in which water emits out of the base dark esteem. When flooding crosswise over two least unites then a dam is built to recognize the limit crosswise over them. This strategy is basically an edge based system [9].

The watershed change more often than not prompts over division of images because of image clamor and other neighborhood inconsistencies. To defeat this, inquires about have proposed numerous methodologies, for example, marker controlled watershed division, various leveled division and multiscale division. Because of number of favorable circumstances watershed change has been generally utilized as a part of many fields of image handling. This strategy is the morphological based image division. This technique create an entire division of the image in isolated area regardless of the possibility that the complexity is poor, consequently maintaining a strategic distance from the requirement for any sort of form joining [10].

Diverse methodologies might be utilized to utilize the watershed rule for division. Neighborhood minima of the slope of the image might be picked as markers, for this situation an over division is delivered. Marker based watershed change makes utilization of particular marker positions which have been either expressly characterized by the client or decided naturally with morphological administrators or different ways.

The watershed change discovers "catchment bowls" and "watershed edge lines" in a image by regarding it as a surface where light pixels are high and dull pixels are low.

\subsection{Similarity Based Segmentation}

In similitude based technique division is expert construct absolutely with respect to the gathering of pixels basically in light of a few capacities.

\subsubsection{Thresholding}

This is the best system in the majority of the division procedures. The strategy is construct absolutely with respect to a limit cost to transform a dark scale image into the paired image.

\subsubsection{Histogram based strategy}

while contrasted with various image graph division strategies histogram-based absolutely techniques regularly require least complex one pass through the pixels so we will educate those are exceptionally proficient because of the reality they. On this strategy, a histogram is processed from the greater part of the pixels inside the image graph.

\section{LITERATURE SURVEY}

\subsection{Clustering Method}

The k-method calculation is an iterative technique this is utilized to segment the image into $\mathrm{k}$-groups. Grouping fundamentally based image division is the way toward allotting a mark to every pixel in a image to such an extent that pixels with the indistinguishable name share positive visual characteristics. In group orientated division parcel the image pixels into bunches. It makes utilization of the multidimensional data. Group orientated approach is a superior approach than histogram-orientated one in sectioning a image, where every pixel has a few properties and is spoken to by a vector.

\subsection{Basic Edge Detection (gradient)}

The image inclination is to discover edge quality and course at area $(\mathrm{x}, \mathrm{y})$ of image, and characterizes as the vector

$$
\nabla f \equiv \operatorname{grad}(f) \equiv\left[\begin{array}{l}
g_{x} \\
g_{y}
\end{array}\right]=\left[\begin{array}{l}
\frac{\partial f}{\partial x} \\
\frac{\partial f}{\partial y}
\end{array}\right]
$$

The magnitude (length) of vector $\nabla f$, denoted as $\mathrm{M}(\mathrm{x}, \mathrm{y})$

$$
\operatorname{mag}(\nabla f)=\sqrt{g_{x}+g_{y}}
$$

The direction of the gradient vector is given by the angle. The heading of an edge at a discretionary point $(\mathrm{x}, \mathrm{y})$ is orthogonal to the bearing. We are managing advanced quantities, so a computerized estimate of the fractional subsidiaries over an area about a point is required.

$$
\alpha(x, y)=\tan ^{-1}\left[\frac{g_{y}}{g_{x}}\right]
$$

All material on each page should fit within a rectangle of $18 \mathrm{x}$ $23.5 \mathrm{~cm}$ (7" x 9.25"), centered on the page, beginning $2.54 \mathrm{~cm}$ (1") from the top of the page and ending with $2.54 \mathrm{~cm}(1 ")$ from the bottom. The right and left margins should be $1.9 \mathrm{~cm}$ (.75"). The text should be in two $8.45 \mathrm{~cm}$ (3.33") columns with a $.83 \mathrm{~cm}(.33 ")$ gutter.

\subsubsection{Roberts Cross-gradient Operators}

\begin{tabular}{|c|c|}
\hline-1 & 0 \\
\hline 0 & 1 \\
\hline
\end{tabular}

(a)

\begin{tabular}{|c|c|}
\hline 0 & -1 \\
\hline 1 & 0 \\
\hline
\end{tabular}

(b)
Fig. 3 Roberts mask

$$
\begin{aligned}
& g_{x}=\frac{\partial f}{\partial x}=\left(z_{9}-z_{5}\right) \\
& g_{y}=\frac{\partial f}{\partial y}=\left(z_{8}-z_{6}\right)
\end{aligned}
$$

\subsubsection{Prewitt operator}

\begin{tabular}{|c|c|c|}
\hline-1 & -1 & -1 \\
\hline 0 & 0 & 0 \\
\hline 1 & 1 & 1 \\
\hline (c) \\
\hline 0 & 1 & 1 \\
\hline-1 & 0 & 1 \\
\hline-1 & -1 & 0 \\
\hline
\end{tabular}

(e)

\begin{tabular}{|c|c|c|}
\hline-1 & 0 & 1 \\
\hline-1 & 0 & 1 \\
\hline-1 & 0 & 1 \\
\hline
\end{tabular}

(d)

\begin{tabular}{|c|c|c|}
\hline-1 & -1 & 0 \\
\hline-1 & 0 & 1 \\
\hline 0 & 1 & 1 \\
\hline
\end{tabular}

(f)
Fig. 4 Prewitt's mask 


$$
\begin{aligned}
& g_{x}=\frac{\partial f}{\partial x}=\left(z_{7}+z_{8}+z_{9}\right)-\left(z_{1}+z_{2}+z_{3}\right) \\
& g_{y}=\frac{\partial f}{\partial y}=\left(z_{3}+z_{6}+z_{9}\right)-\left(z_{1}+z_{4}+z_{7}\right)
\end{aligned}
$$

\subsubsection{Sobel operator}

\begin{tabular}{|c|c|c|}
\hline-1 & -2 & -1 \\
\hline 0 & 0 & 0 \\
\hline 1 & 2 & 1 \\
\hline
\end{tabular}

\begin{tabular}{|c|c|c|}
\hline-1 & 0 & 1 \\
\hline-2 & 0 & 2 \\
\hline-1 & 0 & 1 \\
\hline
\end{tabular}

(e)

\begin{tabular}{|c|c|c|}
\hline 0 & 1 & 2 \\
\hline-1 & 0 & 1 \\
\hline-2 & -1 & 0 \\
\hline
\end{tabular}

(f)

(f)

\begin{tabular}{|c|c|c|}
\hline-2 & -1 & 0 \\
\hline-1 & 0 & 1 \\
\hline 0 & 1 & 2 \\
\hline
\end{tabular}

(g)

Fig. 5 (e)-(g) are the region of an image and various masks used to compute the gradient at the point labeled

$$
\begin{aligned}
& g_{x}=\frac{\partial f}{\partial x}=\left(z_{7}+2 z_{8}+z_{9}\right)-\left(z_{1}+2 z_{2}+z_{3}\right) \\
& g_{y}=\frac{\partial f}{\partial y}=\left(z_{3}+2 z_{6}+z_{9}\right)-\left(z_{1}+2 z_{4}+z_{7}\right)
\end{aligned}
$$

The Sobel cover uses 2 in the center territory for image smoothing. The Prewitt spreads are more direct to execute than Sobel covers, yet the Sobel[2] covers have better racket suppression(smoothing) traits makes them best.

In the past talk, we just look at to get the end. In any case, this utilization is not for the most part charming, so an approach used a great part of the time is to generally the enormity of the slant by out and out qualities:

$$
M(x, y) \approx\left|g_{x}\right|+\left|g_{y}\right|
$$

\subsection{Region Based Segmentation}

Homogeneity resources of the image areas are utilized to segment the image in the division procedure. The idea of local based thoroughly image division is to expand homogeneity in each sectioned local. Homogeneity criteria can be constructing absolutely with respect to the dim level and shading surface of the image graph. Decision of right homogeneity gauges is most basic and effect division execution. District based thoroughly image division might be classified as region developing and place.

1. Change of RGB become flushed plentiful to Gray adjustment redden space. When we catch the data pixel from RGB[3] to Gray adjustment model we can get a greater narrative picture.

2. Process grids Weight and Diagonal (W and D)

3. In the standardized cut modification, we utilize eigen morals and eigenvectors to procurement out the cut in the model that office to obtaining out the commemoration edges in the picture.

4. Unravel the model for tolerating eigenvectors and unique Eigen values.[4]
From the model up high proclaimed we can obtaining out the Eigen morals and eigenvectors.

5. Utilize the eigenvector with the extra native Eigen incentive to bi-segment the diagram. Here to bi segment the information pixel we charge to utilize the eigenvector of the extra native Eigen esteem. The astuteness for this to utilize the extra native is we charge to shorten the Rayleigh gauge so we can truncate the standardized cut. 6 . Recursively portion the unrelieved areas on the off chance that it is essential.

After we cut the area by application standardized slice modification we charge to cut the two areas of the information pixel on the off chance that it is extremely vital to cut. Shape the underneath pipeline unmistakably to begin with, we have to discover the weight between every one of the pixels in the picture then from that weight capacities we have to make a weight network. From that weight network, we can figure the askew components of the corner to corner grid by just basically including the each line in the weight framework. And after that we have to enter those components in the askew position then we can get the inclining grid. At that point from the weight grid and the corner to corner lattice, we have to discover the Covraiance network just by doing W-D.

\section{PROPOSED ALGORITHM}

The Algorithm for this plan is as per the following:-

Step 1. Perused the first picture of size $\mathrm{m} x \mathrm{n}$.

Step 2. Present the edge in $\mathrm{x}$ bearing to get $\mathrm{g}(\mathrm{x}, \mathrm{y}, \mathrm{T})$ or on the other hand we can specifically have the grayscale picture $g$ $(\mathrm{x}, \mathrm{y}, \mathrm{T})$.

It $=\mathrm{g}(\mathrm{x}, \mathrm{y})$ : Initial Image

Step 3. Set $\mathrm{dx}=0.1, \mathrm{dt}=0.1$

Step 4. for $\mathrm{t}=1$ :niterations where $\mathrm{n}=400$

Display the image region with final segmented image. For that Covariance matrix,[5] we need to find out the Eigen values and eigenvectors[8]. And then by taking the eigenvector values of the second smallest Eigen values, we can divide the image into two parts, on the basis of the sign this eigenvector contains. Then after dividing the image into two parts, we can apply the same procedure to the segmented part. Then we can get a better-segmented image [10]

\section{RESULTS ANALYSIS}

Distance from a problematic strategy which blends information pixel to build In the given outcome we have clearly shown how data pixel mixing has been refrained from according to the proposed Algorithm. As per set the limit the last picture divided has taken 400 accentuates yet separated the exact question or substance in the given picture. Our proposed procedure has laid out how the mixing of data pixel mixing augmentation the course of action confuse for substance affirmation of specific challenge .however with the use of component change of edge method this issue can be constrained to give successful results. To perform locale based substance recuperation get ready limit conditions is somehow shown to be more efficient. We proposed to keep up a key. Classification error. 

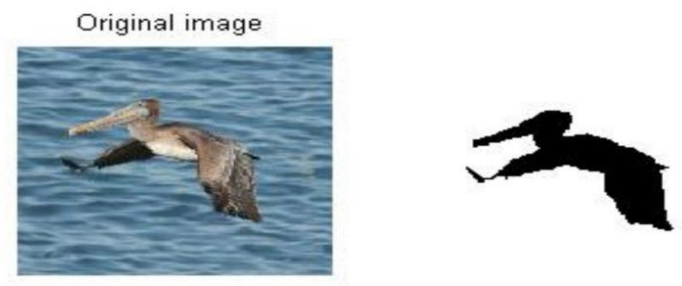

segmented image
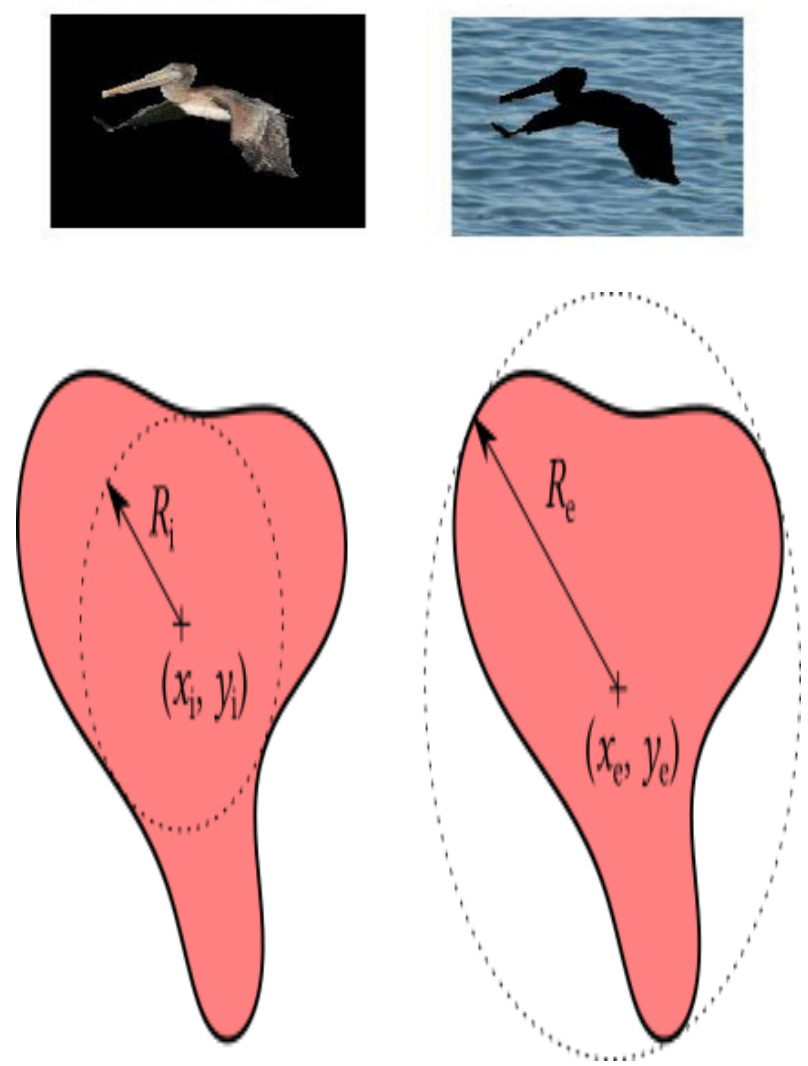

Fig. 6 Coordinates of Segmentation
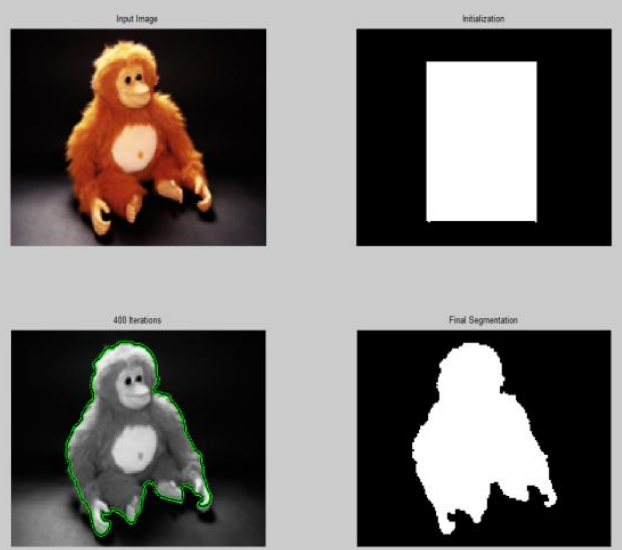

Fig 7. Final Result after 400 Iteration

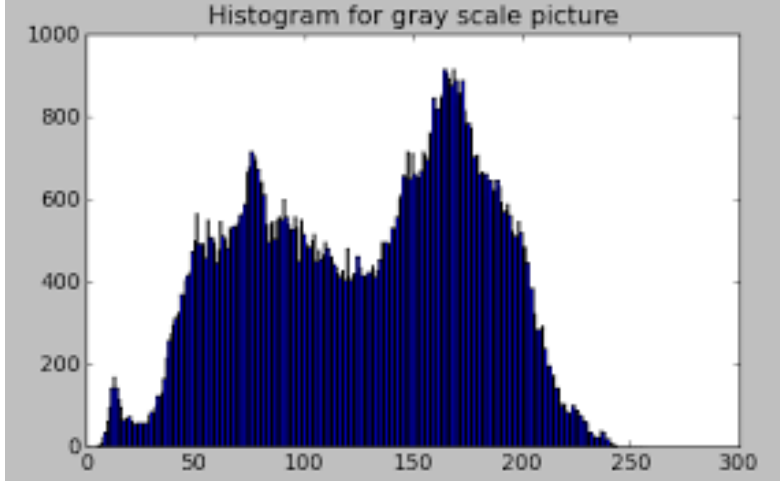

Fig 8. Generated image of histogram of probe image

\section{FUTURE SCOPE \& CONCLUSION}

In this paper, we have described and inspected noteworthy image segmentation computations. image fragmented counts have a promising future ahead since they are the start of image get ready and computer vision and have transformed into the focal point of contemporary research. Despite many years of research, there is no, for the most part, recognized Algorithm segmentation computation. Since image segmentation is affected by clusters of segments, for instance, sort of image, shading, threshold, the level of commotion, and so forth. Thusly there is no single figuring that is germane on an extensive variety of images and nature of the issue. As a result of each and every above component, image segmentation still remains a noteworthy pending issue in the scopes of image planning. In this paper, we propose another region based changed Algorithm for region image segmentation. This method utilizes a false cover to restrain the amount of cycle to meet quickly center a low time for fragmented the image.

\section{REFERENCES}

[1] L. Lazos, R. Poovendran, and J. A. Ritcey, Analytic evaluation of target detection in eterogeneous wireless sensor networks," ACM Trans. Sensor Networks, vol. 5, no. 2, pp. 1-38, March 2013.

[2] J. Jeong, Y. Gu, T. He, and D. Du, "VISA: Virtual Scanning Algorithm for Dynamic Protection of Road Networks," in Proc. of 28th IEEE Conference on Computer Communications (INFOCOM 09), Rio de Janeiro, Brazil, April 2009.Fröhlich, B. and Plate, J. 2000. The cubic mouse: a new device for threedimensional input. In Proceedings of the SIGCHI Conference on Human Factors in Computing Systems

[3] G. Lu, N. Sadagopan, B. Krishnamachari, and A. Goel, "Delay Efficient Sleep Scheduling in Wireless Sensor Networks," in INFOCOM. IEEE, 2005.

[4] Q. Cao, T. Abdelzaher, T. He, and J. Stankovic, "Towards Optimal Sleep Scheduling in Sensor Networks for Rare Event Detection ," in IPSN. ACM/IEEE, 2005.

[5] D. Tian and N. Georganas, "A Node Scheduling Scheme for Energy Conservation in Large Wireless Sensor Networks," Wireless Communications and Mobile Computing Journal, May 2011.

[6] C. Gui and P. Mohapatra, "Power Conservation and Quality of Surveillance in Target Tracking Sensor Networks," in MOBICOM. Philadelphia, PA, USA: ACM, Sep. 2004.

[7] M. Mar 'oti, B. Kusy, G. Simon, and 'AkosL'edeczi, 
"The Flooding Time Synchronization Protocol," in SENSYS. Baltimore, Maryland, USA: ACM, Nov. 2004.

[8] L. Lazos, R. Poovendran, and J. A. Ritcey, Analytic evaluation of target detection in eterogeneous wireless sensor networks," ACM Trans. Sensor Networks, vol. 5, no. 2, pp. 1-38, March 2013.

[9] J. Hwang, T. He, and Y. Kim, "Exploring In-Situ Sensing Irregularity in Wireless Sensor Networks," in SENSYS. ACM, Nov. 2014, pp. 289-303.

[10] Y. Gu and T. He, "Data Forwarding in Extremely Low Duty-Cycle Sensor Networks with Unreliable Communication Links," in SENSYS. Sydney, Australia: ACM, Nov. 2007, pp. 321-334.

[11] J.Shi and J.Malik, "Normalized cuts and image segmentation "IEEE Trans. pattern Anal. Mach. Intell.
Vol. 22, no.8, pp. 888-905,Aug 2000

[12] W.Tao,H. Jin, and Y. Zhang,"Color image segmentation based on mean shift and normalizedcuts,"IEEE Trans.Syst.,Man, Cybern. B, Cybern., vol.37,no.5, pp. 1382-1389, Oct.2007.

[13] W.Tao, H.Jin, and Y. Zhang "Image Thresholding using Graph cut "IEEE Trans.pattern And Vol 38,No5,pp.1181-1195,Sep2008

[14] http://en.wikipedia.org/wiki/Graph-cut

[15] http: // en. Wikipedia .org / wiki / Graph _ cuts _ in _ computer_vision

[16] Comaniciu, D. and Meer, P. 1997. Robust analysis of feature spaces: Color image segmentation. In Proceedings of IEEE Conference on Computer Vision and Pattern Recognition, pp.750-755. 